\title{
High-Precision Determination of the Neutron Coherent Scattering Length
}

\section{Apoorva G. Wagh and Sohrab Abbas}

86 Dhruva, Solid State Physics Division, Bhabha Atomic Research Centre, Mumbai 400085, India

\author{
The neutron coherent scattering length $b_{\mathrm{c}}$ \\ has been determined interferometrically \\ to an uncertainty of about $5 \times 10^{-5}$ by \\ measuring the nondispersive phase. We \\ propose improving the uncertainty to \\ about $10^{-6}$ by optimizing various para- \\ meters of the interferometric experiment. \\ Any uncertainty in the $b_{\mathrm{c}}$ determination \\ arising from possible variations in the \\ constitution of the ambient air can be \\ eliminated by performing the experiment \\ in vacuum. When such uncertainty is \\ attained, it becomes necessary to account \\ for the neutron beam refraction at the \\ sample-ambient interfaces, to infer the
}

correct $b_{\mathrm{c}}$ from the observed phase. The formula for the phase used hitherto is approximate and would significantly overestimate $b_{c}$. The refractive index for neutrons can thus be determined to a phenomenal uncertainty of about $10^{-12}$.

Key words: coherent scattering length; neutron interferometry; nondispersive phase.

Accepted: August 11, 2004

Available online: http://www.nist.gov/jres

\section{Introduction and Discussion}

The coherent scattering length for neutrons is an important parameter that describes the neutron-nuclear interaction. A precise knowledge of coherent scattering length is important for understanding the basic nucleon-nucleon interaction, charge independence and charge symmetry of the nuclear forces. Precise determination of $b_{\mathrm{c}}$ is also needed for different isotopes for material science applications [1,2]. There are many techniques such as Christiansen filter, gravity refractometer, mirror reflection, prism reflection, pendellösung oscillation etc. $[1,2]$ for $b_{c}$ determination. Shull et al. [3] attained the least uncertainty of about $0.03 \%$ in $b_{\mathrm{c}}$ determination of silicon by observing the pendellösung oscillations.

Perfect crystal interferometry affords precise determination of the coherent scattering length [1-2] of samples. With a parallel-faced sample slab of thickness $D$ and atomic density $N$, placed normal to one subbeam in the interferometer, neutrons acquire the phase

$$
\Phi=-\left(N b_{\mathrm{c}}-N_{\mathrm{a}} b_{\mathrm{a}}\right) D \lambda .
$$

Here $\lambda$ denotes the incident neutron wavelength and symbols with the subscript a stand for the corresponding properties of ambient air or vacuum. This variation of $\Phi$ with $\lambda$ over the spread $\Delta \lambda$ in the incident wavelengths reduces the interference contrast. The consequent loss in phase precision limits the attainable $b_{c}$ uncertainty to about $10^{-3}$.

Rauch et al. [4] reduced the uncertainty to about $4.7 \times 10^{-4}$ by following Scherm's suggestion to insert the sample with its surface parallel to the Bragg planes of the interferometer. Neutrons of each wavelength from the beamsplitter are then incident at the corresponding Bragg angle $\theta_{\mathrm{B}}$ to the sample and the phase

$$
\Phi \approx-\left(N b_{\mathrm{c}}-N_{\mathrm{a}} b_{\mathrm{a}}\right) D \lambda / \sin \theta_{\mathrm{B}}=-2\left(N b_{\mathrm{c}}-N_{\mathrm{a}} b_{\mathrm{a}}\right) D d,
$$

is nondispersive. Here $d$ symbolizes the Bragg planar spacing. However, here the phase varies sharply with the inclination $\theta$ of the sample (cf. $\Phi_{0-\mathrm{I}}$ and $\Phi_{\mathrm{II}-0}$ curves in Fig. 1). The nondispersivity condition therefore requires the sample to be aligned with arcsecond precision. 


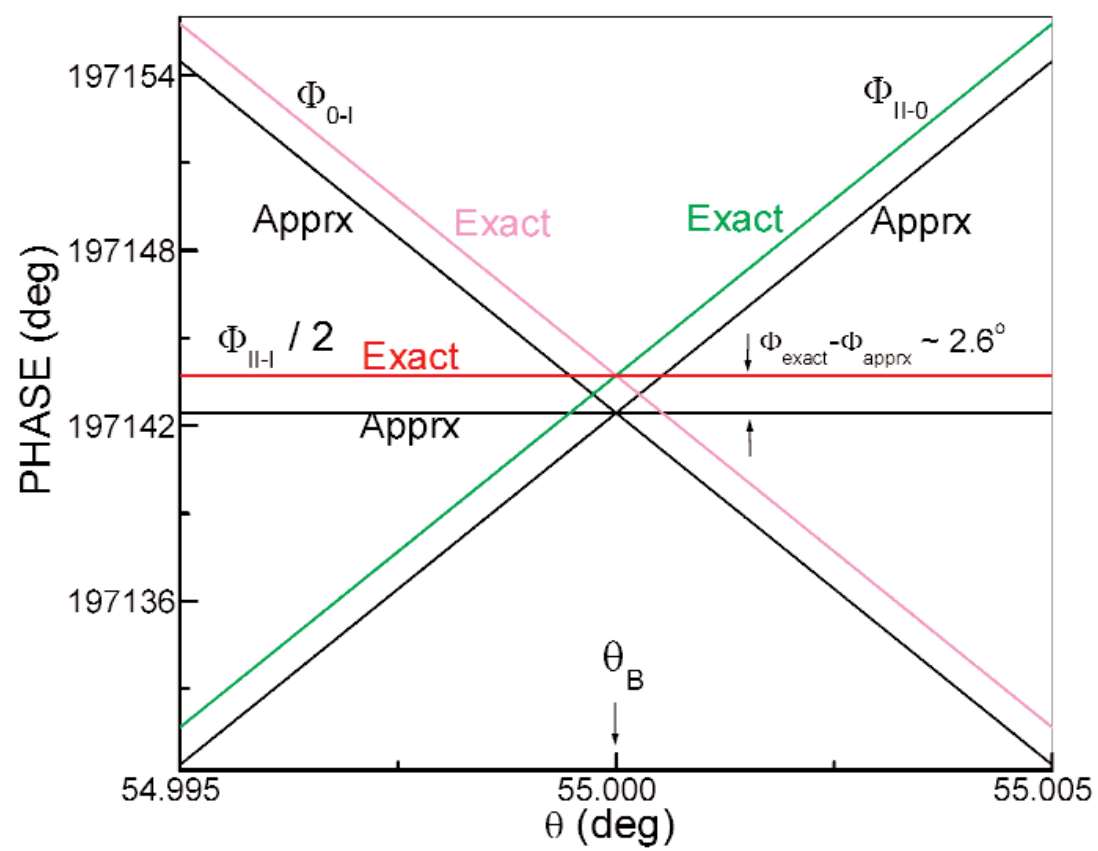

Fig. 1. Exact and approximate nondispersive phases in air.

Ioffe et al. [5] overcame this limitation by measuring the phase shift between interferograms recorded with the sample placed alternately in subbeams I and II (Fig. 2). This eliminates the first order variation of the phase (cf. $\Phi_{\mathrm{II}-\mathrm{I}}$ curve in Fig. 1) with the horizontal misalignment $\Delta \theta$ from $\theta_{\mathrm{B}}$. The sample alignment thus requires only arcminute precision to locate the minimum in $\Phi_{\mathrm{II-}}$, occurring at the intersection of $\Phi_{0-\mathrm{I}}$ and $\Phi_{\mathrm{II}-0}$ curves. The nondispersive phase shift

$$
\Phi_{\mathrm{I}-\mathrm{II}} \approx-\frac{\left(N b_{\mathrm{c}}-N_{\mathrm{a}} b_{\mathrm{a}}\right) D d}{\cos \Delta \gamma} 2\left(2+(\Delta \theta)^{2}\left\{1+2 \cot ^{2} \theta_{\mathrm{B}}\right\}\right),
$$

then determines the coherent scattering length

$$
b_{\mathrm{c}} \approx-\frac{\Phi_{\mathrm{I}-\mathrm{I}} \cos \Delta \gamma}{4 N D d\left(1+\frac{\Delta \theta^{2}}{2}\left(1+2 \cot ^{2} \theta_{\mathrm{B}}\right)\right)}+\frac{N_{\mathrm{a}} b_{\mathrm{a}}}{N},
$$

$\Delta \gamma$ denoting the vertical misalignment of the sample. The experiment [5] achieved a precision $\Delta b_{\mathrm{c}} / b_{\mathrm{c}}$ of $5.1 \times 10^{-5}$, whose source-wise constituents are listed on the left hand side of Table 1. By far the most predominant contribution arises from the relative variation $\Delta D / D$ in the sample thickness.

The uncertainty can hence be lowered by increasing $D$ and reducing its variation $\Delta D$. An increase in $D$ dictates a large Bragg angle (Fig. 3). For practical reasons, we limit $\theta_{\mathrm{B}}$ to $55^{\circ}$ (Fig. 2) allowing $D=26.5 \mathrm{~mm}$ for a $3 \mathrm{~mm}$ wide incident neutron beam.
The width of the interferometer becomes rather large, about $12.5 \mathrm{~cm}$, at this $\theta_{\mathrm{B}}$. Attaining $\Delta D=0.1 \mu \mathrm{m}$ with a precision grinding and polishing machine would yield about an order of magnitude reduction in the $\Delta D / D$ contribution to $\Delta b_{\mathrm{c}} / b_{\mathrm{c}}$. In addition, the phase also increases by the same factor as $D$, reducing the $\Delta \Phi / \Phi$ contribution. The corresponding neutron path length of $32.4 \mathrm{~mm}$ within the sample would still yield a good interference contrast as observed by Rauch et al. [4]. Further, we can maximize $d$ to $0.314 \mathrm{~nm}$ by choosing the $\{111\}$ Bragg reflection for the interferometer (hence $\lambda=0.514 \mathrm{~nm}$ ) to further enhance $\Phi$ and reduce $\Delta \Phi / \Phi$. A thermal enclosure around and vibration isolation of the interferometer reduces the phase drift to a fraction of a degree over a day [2,5]. The effect of this phase drift over a typical measurement duration of a few hours, is minimized by recording the $\mathrm{O}$ and $\mathrm{H}$ detector intensities (Fig. 2) for the three positions (I, II, and Out) of the sample in succession at each angular setting of the phase flag. A phase error of about $0.3^{\circ}$, thus routinely achieved in interferometric experiments, is included in Table 1. The contribution from the uncertainty in the refractive index of air, dependent on variations in the temperature, pressure and relative humidity, can be larger than that assumed in [5], viz., $N_{\mathrm{a}} b_{\mathrm{a}} / N=(9.137 \pm 0.009) \times 10^{-3} \mathrm{fm}$. This can be eliminated by performing the experiment in vacuum. With a crystalline silicon sample $\left(N d=1.57 \times 10^{15} \mathrm{~cm}^{-2}\right)$, our proposed phase $\Phi_{\mathrm{I}-\mathrm{II}}=-394284.8^{\circ}$ will yield $b_{\mathrm{c}}$ with uncertainties as shown on the right hand side of Table 1. 


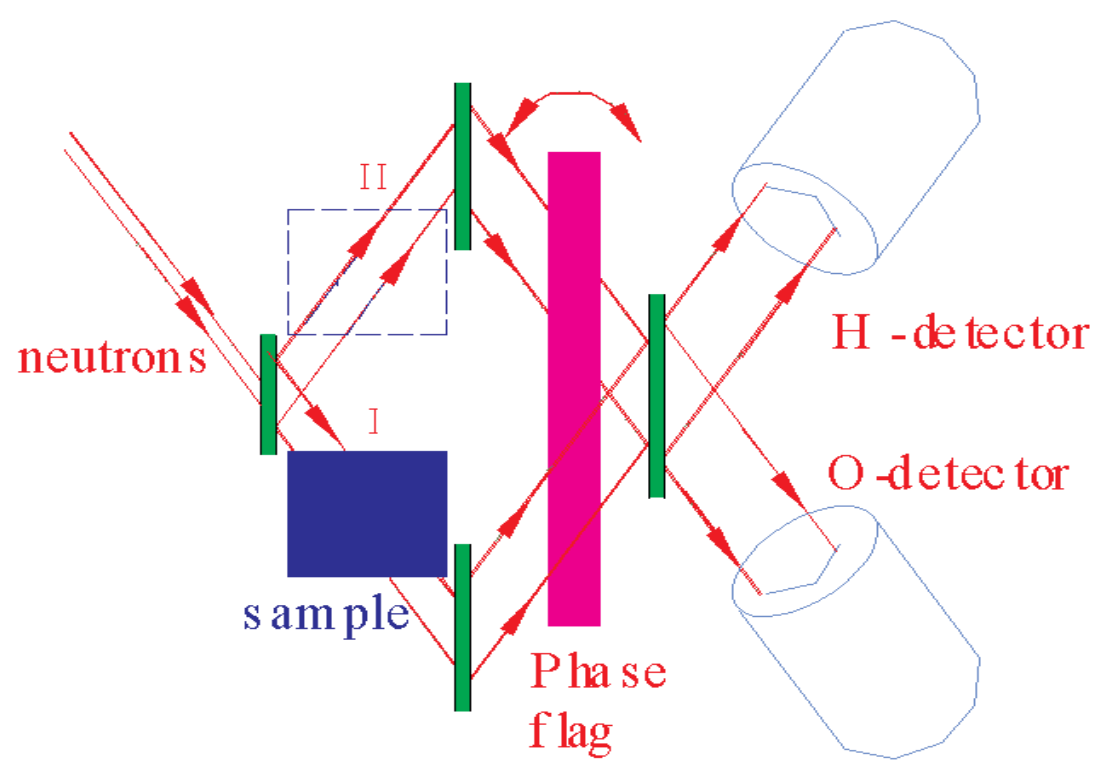

Fig. 2. Our proposal.

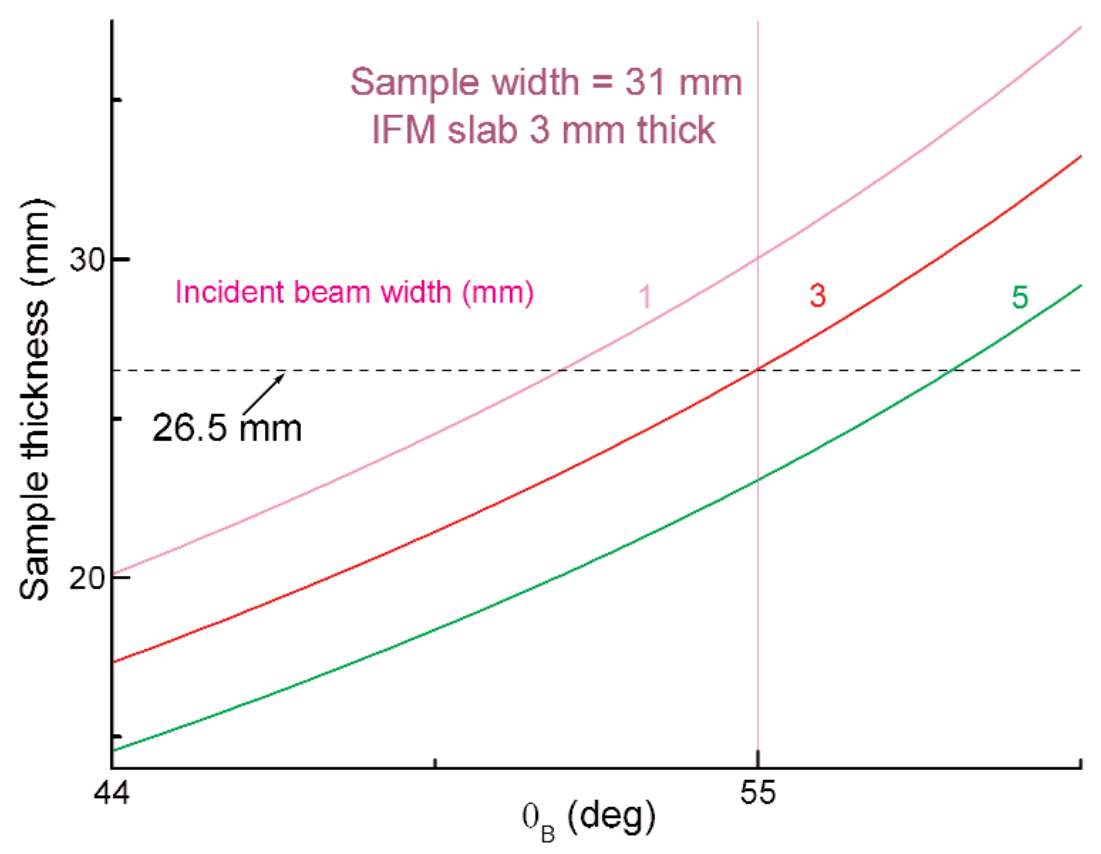

Fig. 3. Variation of the allowed sample thickness with Bragg angle. 
Table 1. Comparison between various $\Delta b_{\mathrm{c}} / b_{\mathrm{c}}$ contributions at present [5] and in the proposed experiment

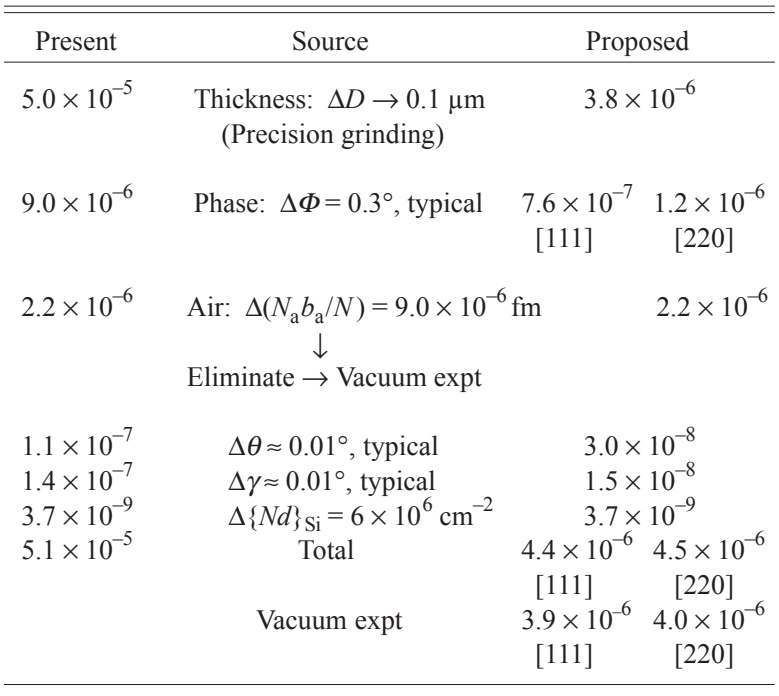

When such uncertainty is achieved, it becomes necessary to account for neutron refraction at the ambient sample interfaces. Conservation of the tangential component of the neutron wave vector across the interface yields the exact phase

$$
\begin{aligned}
\Phi_{\text {I-II }} & =\frac{4 \pi D}{\lambda}\left(\sqrt{n^{2}-n_{\mathrm{a}}^{2} \cos ^{2}} \theta_{\mathrm{B}}-n_{\mathrm{a}} \sin \theta_{\mathrm{B}}\right) \\
& =4 \pi D\left(\sqrt{\frac{-\left(N b_{\mathrm{c}}-N_{\mathrm{a}} b_{\mathrm{a}}\right)}{\pi}+\frac{n_{\mathrm{a}}^{2}}{4 d^{2}}}-\frac{n_{\mathrm{a}}}{2 d}\right),
\end{aligned}
$$

$n$ denoting the refractive index. The exact and approximate [Eq. (3)] phases for $\Delta \gamma=0$ in our proposal are plotted in Fig. 1. The exact phase is greater by about $2.6^{\circ}$ at $\theta=\theta_{\mathrm{B}}$. The exact phase [Eq. (5)] is rigorously nondispersive only in vacuum, i.e., when $n_{\mathrm{a}}=1$. However, since the refractive index of air differs from unity only by about $1.4 \times 10^{-8}$, the phase is nondispersive to an excellent approximation even in air, to better than $3 \times 10^{-10}$ for an incident wavelength spread $\Delta \lambda / \lambda$ of $1 \%$. Equation (5) yields the coherent scattering length

$$
b_{\mathrm{c}}=\frac{n_{\mathrm{a}} \Phi_{\mathrm{I}-\mathrm{II}}}{4 N D d}-\frac{\Phi_{\mathrm{I}-\mathrm{II}}^{2}}{16 \pi N D^{2}}+\frac{N_{\mathrm{a}} b_{\mathrm{a}}}{N} .
$$

Therefore the correction to the inferred $b_{\mathrm{c}}$ due to the refraction effects

$$
\frac{\Delta b_{\mathrm{c}}}{b_{\mathrm{c}}} \approx-\frac{N b_{\mathrm{c}} d^{2}}{\pi}=-6.5 \times 10^{-6},
$$

slightly exceeds the proposed precision in magnitude, underscoring the importance of refraction effects.
The refractive index, $n=\left(1-N b_{c} \lambda^{2} / \pi\right)^{1 / 2}$ of silicon for thermal neutrons equals unity to within about $1 \times 10^{-6}$. Our proposal can thus determine the refractive power, $n-1 \approx 10^{-6}$, with a relative uncertainty of about $10^{-6}$, and hence the refractive index to a phenomenal uncertainty of about $10^{-12}$.

In conclusion, we have proposed an optimized interferometric measurement of the nondispersive phase to determine the neutron coherent scattering length of silicon to an uncertainty of $4 \times 10^{-6}$.

\section{References}

[1] L. Koester, H. Rauch, and E. Seymann, Atom. Data Nucl. Data Tables 49, 65 (1991).

[2] H. Rauch and S.A. Werner, Neutron Interferometry, Oxford University Press, New York (2000).

[3] C. G. Shull and J. A. Oberteuffer, Phys. Rev. Lett. 29, 871 (1972).

[4] H. Rauch, E. Seidl, D. Tuppinger, D. Petrascheck, and R. Scherm, Z. Phys. B 69, 313 (1987).

[5] A. Ioffe, D. L. Jacobson, M. Arif, M. Vrana, S. A. Werner, P. Fischer, G. L. Greene, and F. Mezei, Phys. Review A 58, 1475 (1998).

About the authors: Prof. A. G. Wagh and S. Abbas are physicists at the Solid State Physics Division of Bhabha Atomic Research Centre in Mumbai (India), working on neutron interferometry and geometric phase. 